\title{
Comportamiento de Corrosión-Erosión en Recubrimientos de TiN/ZrN Depositados sobre Acero AISI 4140
}

\author{
Willian Aperador ${ }^{(1)}$, Aura S. Mejía ${ }^{(2)}$ y Julio Caicedo ${ }^{(3)}$ \\ (1) Universidad Militar Nueva Granada, Departamento de Ingeniería, Carrera 11 No. 101-80, \\ Bogotá-Colombia. (e-mail: g.ing.materiales@gmail.com) \\ (2) Escuela Colombiana de Ingeniería Julio Garavito, Facultad de Ciencias, Bogotá-Colombia. \\ (3) Universidad del Valle, Escuela de Materiales, Ciudad Universitaria, Meléndez, A. A. 25360 Cali- \\ Colombia
}

Recibido May. 23, 2013; Aceptado Jul. 31, 2013; Versión final recibida Ago. 6, 2013

\begin{abstract}
Resumen
Se depositaron películas delgadas de nitruro de titanio/nitruro de zirconio sobre acero AISI 4140 mediante un sistema multi-blanco magnetrón pulverización (sputtering) con radiofrecuencia. El proceso se desarrolló bajo el sistema de rotación del sustrato que permitió situar el sustrato de frente a los blancos de titanio y zirconio, generando la super-red con periodos de 1, 50, 100 y 200 bicapas. Las multicapas fueron evaluadas frente a la sinergia corrosión-erosión en una solución compuesta por $\mathrm{NaCl}$ al $3 \%$ y sílice. La caracterización electroquímica se realizó mediante la técnica de espectroscopía de impedancia electroquímica. La superficie fue evaluada posterior al ensayo mecánico y electroquímico mediante la técnica de microscopia electrónica de barrido. Por medio del análisis de desempeño se observó que el material con mayor efecto sinérgico fue el sustrato. También se muestra que al aumentar las bicapas en el sistema nitruro de titanio/nitruro de zirconio el fenómeno corrosivo controla el deterioro del material.
\end{abstract}

Palabras clave: multicapas, pulverización, corrosión, erosión, desgaste, recubrimientos

\section{Corrosion-Erosion Behavior in Coatings of TiN / ZrN Deposited on AISI 4140 Steel}

\begin{abstract}
Thin films of titanium nitride/zirconium nitride were deposited on AISI 4140 steel by a multi-target magnetron sputtering system with radio frequency. The process was developed under the substrate rotation system which allowed placing the substrate against the titanium and zirconium targets, generating the super-lattice with periods of $1,50,100$, and 200 bilayers. The multilayers were evaluated against the corrosion-erosion synergy in a solution composed by $3 \% \mathrm{NaCl}$ and silica. The electrochemical characterization was performed by electrochemical impedance spectroscopy. After the mechanic and electrochemical tests the surface was evaluated using scanning electron microscopy. By performance analysis it was observed that the material with higher synergic effect was the substrate. It is also shown that by increasing the bilayers in the system titanium nitride/zirconium nitride the corrosive phenomenon controls the material deterioration.
\end{abstract}




\section{INTRODUCCIÓN}

En la actualidad, gracias al fenómeno de la globalización, el campo de acción para comercializar productos, bienes y servicios se ha ampliado (Rajahram et al., 2009). Como consecuencia, los niveles de competitividad han aumentado y esto obliga a las empresas a encontrar un mejoramiento continuo dentro de su proceso productivo el cual les permita aumentar su producción con una excelente calidad y a menores costos (Farzad y Jingli., 2011; López et al., 2011). La industria manufacturera utiliza herramientas o dispositivos como las motobombas, las cuales se desgastan y corroen rápidamente debido a las condiciones de operación a las que trabaja, originando altos costos económicos. Para evitar el deterioro precario de los componentes de las motobombas se ha optado por fabricar sus partes con una amplia gama de materiales tales como aceros inoxidables, níquel y caucho (Neville y Wang., 2009), sin embargo, es de gran importancia desarrollar sistemas multicapas que igualen o superen las propiedades mecánicas y el comportamiento frente a la corrosión, en particular de los aceros inoxidables, con materiales de menor costo y mayor vida útil funcional.

Gracias al surgimiento y evolución de las técnicas de procesamiento por plasma, en especial la deposición física en fase vapor ó PVD como es conocida por sus siglas en inglés, se han logrado desarrollar desde hace muchos años, materiales que obtenidos a unos espesores de baja dimensión (del orden de las micras) permiten modificar las propiedades superficiales de la pieza, conservando las propiedades del material sobre el cual son depositados, combinación que causa en una mejora en el desempeño durante las condiciones usuales de trabajo (Philip et al., 1999; Xi y Scott 1995). Entre los materiales que son fabricados mediante los procesos de PVD se tienen los materiales binarios como: el TiN, el $\mathrm{CrN}$, el $\mathrm{ZrN}$; ternarios como: el TiCN, el TiAIN, el AITiN; y cuaternarios como: TiAICrN, TiAIVN, así como combinaciones de multicapas de estos para mejorar sus propiedades mecánicas como son la dureza y la tenacidad (Barshilia et al., 2007; Barshilia et al., 2009).

Actualmente, se han desarrollados cuatro recubrimientos a nivel experimental (AICrN, [TiN/ZrN]n, [TiAIN/TiAINbN]n y $\mathrm{B}_{4} \mathrm{C} / \mathrm{BCN} /\{\mathrm{BCN} / \mathrm{h}-\mathrm{BN}\} \mathrm{n} / \mathrm{c}-\mathrm{BN}$ ) que presentaron buenas propiedades de resistencia a la corrosión y al desgaste, pero que no han sido validados mediante pruebas de mecanizado para evaluar su desempeño en aplicaciones industriales de este tipo (Nieto et al., 2010; Junhua et al., 2010; Philip et al., 1999).

El desgaste por erosión, entendido como el daño provocado por el impacto de partículas duras presentes en un fluido, afecta equipos como turbinas, bombas, tuberías y otros dispositivos que transportan fluidos y los cuales pueden estar sometidos a cambios bruscos de presión, provocando efectos como erosión de superficies sólidas, vibraciones, ruidos excesivos, disminución de eficiencia y otros, lo que finalmente causa enormes gastos por reparo o reposición de este tipo de equipos (Caicedo et al., 2010). Adicionalmente estas piezas que están expuestas a la acción de estos fluidos pueden presentar procesos de corrosión por erosión disminuyendo de manera acelerada su vida útil. La corrosión por erosión es una aceleración en la velocidad de corrosión de un metal debido al movimiento relativo de un fluido corrosivo y la superficie de un metal (Aperador et al., 2010), si además el fluido presenta contenido de partículas sólidas en suspensión se tiende a incrementar el efecto erosivo que causa el deterioro del metal. Actualmente los recubrimientos duros como los nitruros basados en metales de transición depositados mediante técnicas como la deposición física de vapor y sobre diversos sustratos de acero, se están convirtiendo en la solución de muchos problemas de ingeniería y entre ellos la corrosión, debido a su inercia química (Caicedo et al., 2012; Aperador et al 2011).

El propósito de este trabajo es estudiar el desempeño de los mecanismos de desgaste de los recubrimientos multicapa tipo [TiN/ZrN] $]_{\mathrm{n}}$, los cuales ofrecen protección a sustratos de uso industrial como el acero AISI 4140. Con el fin de analizar su respuesta con la variación del número de bicapas. Este estudio permite determinar el efecto sinérgico entre la corrosión y la erosión, y establecer el efecto protector de los recubrimientos.

\section{METODOLOGÍA}

Se depositaron multicapas de [TiN/ZrN] $]_{n}$ sobre sustratos de acero AISI 4140 (diámetro $2 \mathrm{~cm}$; espesor $4 \mathrm{~mm}$ ) y Si (orientación (100); ). Los recubrimientos se obtuvieron mediante la técnica del magnetrón sputtering multi-blanco en r.f (13.56 MHz). Para la deposición de los recubrimientos se utilizaron blancos de 4 pulgadas de diámetro de $\mathrm{Ti}$ y $\mathrm{Zr}$ con una pureza del 99,9\%. La presión base al interior de la cámara de vacío fue de $4,0 \times 10^{-6}$ mbar. Antes de iniciar la deposición los sustratos fueron sometidos a una limpieza por plasma durante 20 minutos en atmósfera de $\mathrm{Ar}$ a un bias de $-400 \mathrm{~V}$ en r.f. Durante el crecimiento, los gases de trabajo fueron una mezcla de $\operatorname{Ar}(93 \%)$ y $\mathrm{N}_{2}(7 \%)$ con una presión total de trabajo de $4 \times 10^{-2}$ mbar a una temperatura del sustrato de $250^{\circ} \mathrm{C}$ y un bias r.f del sustrato de $-100 \mathrm{~V}$ y una potencia de $350 \mathrm{~W}$. Para la 
deposición de las multicapas el blanco de titanio, como el de zirconio fue periódicamente cubiertos por el obturador de forma alternante, mientras el sustrato se mantenía bajo rotación circular en frente de los blancos para facilitar la formación de los recubrimientos.

Con el fin de estudiar la influencia de la sinergia entre la corrosión dinámica, erosión y corrosión erosión, de recubrimientos tipo multicapas, se depositaron sistemas de $[\mathrm{TiN} / \mathrm{ZrN}]_{n}$ controlando los tiempos de apertura y cierre del obturador. El espesor de los recubrimientos fue obtenido mediante un perfilómetro DEKTAK 8000 con un diámetro de punta de $12 \pm 0,04 \mu \mathrm{m}$ a una longitud de barrido entre $1000 \mu \mathrm{m}-1200 \mu \mathrm{m}$. Para la muestra de 200 bicapas, el espesor fue de 3,00 $\pm 0,04 \mu \mathrm{m}$, y dado que los recubrimientos fueron obtenidos bajo los mismos parámetros de crecimiento y tiempo total de deposición (3 horas), es posible afirmar que los sistemas multicapas tienen un espesor alrededor de este valor.

En cuanto a la evaluación de la resistencia a la corrosión erosión se utilizó un equipo de incidencia de chorro de partícula, como se muestra en la figura 1. Este consiste en un flujo acuoso que se mantiene en movimiento constante por un sistema de recirculación conformado por mangueras, acoples y una bomba de conducción magnética la cual controla la velocidad del flujo, la temperatura del fluido es regulada por medio de una resistencia térmica. La cámara de acrílico permite tener las condiciones adecuadas para realización de los ensayos por ataque directo o por inmersión, y el ángulo de incidencia o ángulo de impacto es seleccionado mediante la posición del porta probeta. El equipo permite controlar tres variables independientemente las cuales son la temperatura del fluido, velocidad del flujo y el ángulo de impacto. Los ensayos se realizaron con un ángulo de impacto de $90^{\circ}$. La velocidad de impacto genera una velocidad lineal promedio de la partícula de $18,5 \mathrm{~m} \mathrm{~s}^{-1}$. El equipo tiene adaptado el electrodo de referencia - ER $(\mathrm{Ag} / \mathrm{AgCl})$, el contraelectrodo -EA (Alambre de platino) y el portamuestras -ET con una área de exposición de la muestra de $1 \mathrm{~cm}^{2}$

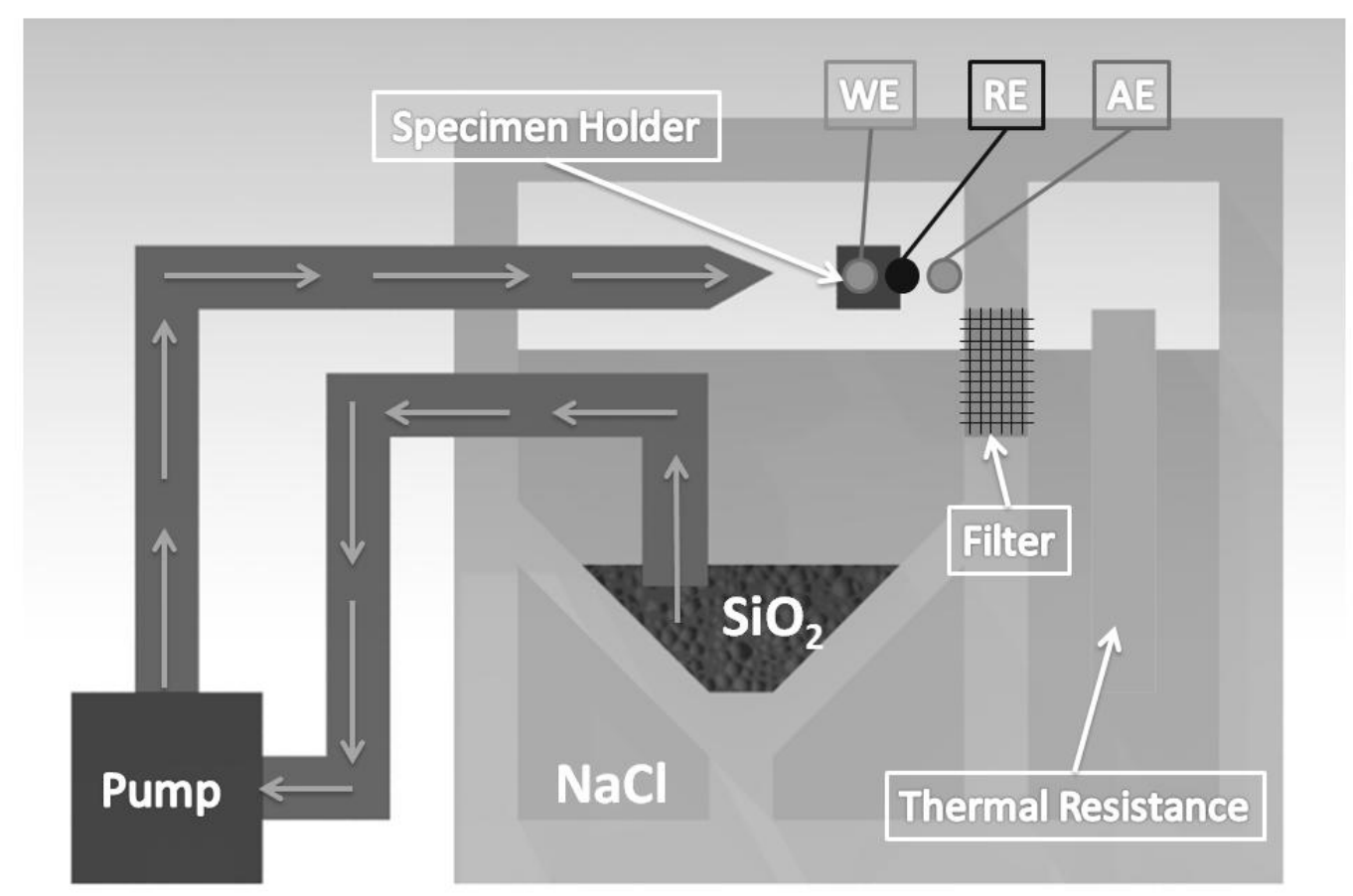

Fig. 1. Esquema de dispositivo empleado en las pruebas de desgaste por corrosión-erosión, EA: Electrodo auxiliar, ET: Electrodo de trabajo, ER: Electrodo de referencia.

Para la evaluación de la resistencia a la corrosión dinámica y corrosión-erosión se utilizó un potenciostato galvanostato Gamry modelo PCl-4 empleando la técnica de espectroscopía de impedancia electroquímica (EIS). Se ubicaron las probetas bajo inmersión en una solución de $\mathrm{NaCl}$ 0,5 M preparada con agua destilada y se adicionaron partículas de sílice $\left(\mathrm{SiO}_{2}\right)$ con tamaño de partícula entre $210 \mu \mathrm{m}$ y $300 \mu \mathrm{m}$ bajo una proporción del $20 \%$ wt con respecto al medio electrolítico. La celda compuesta por un contraelectrodo (Alambre de platino), un electrodo de referencia de $\mathrm{Ag} / \mathrm{AgCl}$ y como electrodo de trabajo se utilizó el acero AISI 4140 sin recubrir y recubierto con multicapas. Los diagramas de Nyquist se obtuvieron con barridos de frecuencia entre $100 \mathrm{kHz}$ y $0,001 \mathrm{~Hz}$ empleando una amplitud de la señal sinusoidal de $10 \mathrm{mV}$ y área expuesta de $1 \mathrm{~cm}^{2}$. La norma utilizada en los criterios de medición y cálculos corresponden a las ASTM G3. Adicionalmente las muestras fueron sometidas a desgaste por erosión durante un tiempo total de exposición 
de 1440 minutos a temperatura de $25^{\circ} \mathrm{C}$ en una solución de $\mathrm{NaCl} 0,5 \mathrm{M}$ con partículas de sílice $\left(\mathrm{SiO}_{2}\right)$. Durante la prueba de inmersión en una solución de $\mathrm{NaCl} 0,5 \mathrm{M}$ con la presencia de partículas de sílice $\left(\mathrm{SiO}_{2}\right)$. Para determinar la pérdida de peso debido a la erosión, las muestras se retiraron de la solución a intervalos de tiempo de 15 minutos, se limpiaron con un chorro de agua, se secaron con aire caliente y se pesaron en una balanza de precisión $(0,1 \mathrm{mg})$. La evaluación del fenómeno erosivo se realizó utilizando protección catódica de $1 \mathrm{~V}$ con respecto al potencial de circuito abierto del acero y el recubrimiento respectivamente de acuerdo con la norma ASTM G 119-93, esta protección catódica garantizó daño superficial sólo por efecto de las partículas erosivas. Los fenómenos de degradación fueron observados con un microscopio electrónico de barrido (SEM), para este proceso se efectuó un corte de la sección transversal de las muestras con una punta de diamante, la cual permitió analizar mediante SEM, las características de crecimiento de las multicapas. Tanto la morfología de crecimiento como las características superficiales, se determinaron con un SEM Phenom FEl equipado con una luz óptica con un rango de magnificación de 525-25,000X.

\section{RESULTADOS Y DISCUSIÓN}

\section{Microscopia electrónica de barrido (SEM)}

La superficie morfológica y el crecimiento columnar de los recubrimientos duros depositadas silicio (Si) fueron estudiadas por medio de la microscopia electrónica de barrido . La figura 2a muestra una micrografía para una bicapa de [TiN/ZrN] y en la figura $2 b$ se indica una micrográfica para la multicapa que incluye 200 bicapas, en la cual puede ser observada la microestructura columnar típica de la película de estos dos tipos de sistemas nanoestructurados. Esta microestructura columnar es debida al continuo bombardeo en el momento del crecimiento la película generado por los átomos neutrales, iones y moléculas que colisionan y compactan la película acercándola la densidad del material en bloque, lo cual es evidenciado por el tamaño de grano, que disminuyen con el bias. Entonces se demuestra que el tamaño y los defectos en el grano decrecen cuando la película se crece aplicando un alto voltaje bias, en valores cercanos a -100V.

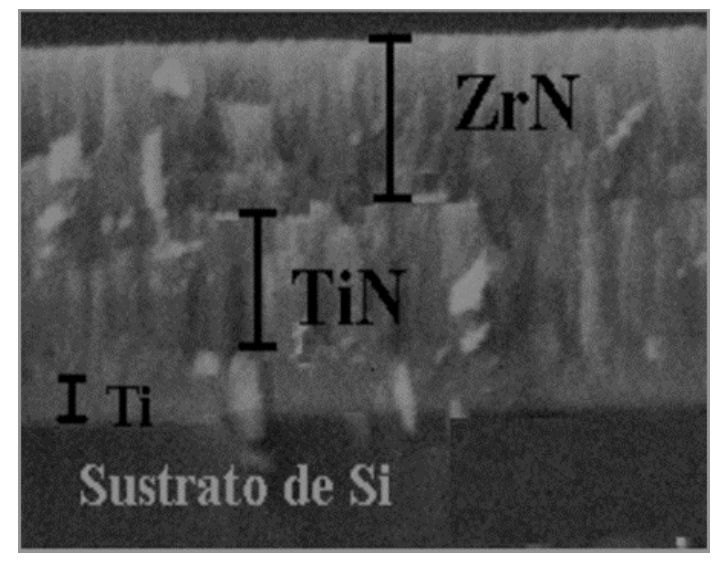

(a)

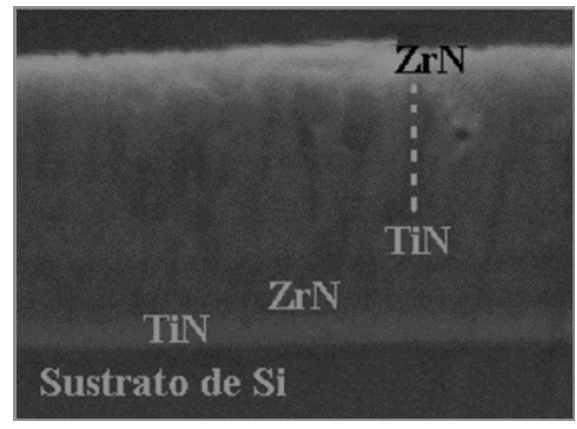

(b)

Fig. 2. Micrografía SEM de la película de [TiN/ZrN]: a) morfología de la microestructura tipo columnar para una bicapa de [TiN/ZrN] con una capa bufer de Ti, (b) morfología de la microestructura tipo columnar para una multicapa de $[\mathrm{TiN} / \mathrm{ZrN}]_{100}$.

\section{Erosión}

En la figura 3, se muestran las diferentes curvas dinámicas de desgaste de los materiales de estudio sometidos a degradación en un medio fluido erosivo constituido con partículas duras de sílice se diferencia con claridad la severidad que presenta el desgaste con respecto a la variación de la bicapas depositadas, en donde se obtiene que al aumentar el valor de las bicapas se obtienen menores pérdidas de espesor del material en comparación con el material base. Este tipo de desgaste observado, el cual es resultado del impacto sucesivo de partículas abrasivas sobre la superficie del material a una velocidad lineal promedio de $18,5 \mathrm{~m} \mathrm{~s}^{-1}$, muestra la clara dependencia con el ángulo de impacto debido a las condiciones energéticas que envuelven el impacto de la partícula sobre la superficie del material. Adicionalmente, con la aplicación al sustrato de los recubrimientos multicapas se obtiene una disminución en la inclinación de las curvas hacia valores más bajos de pérdida de peso con el tiempo, mostrando el efecto benéfico de recubrir el acero 4140. Por su parte la multicapa con 200 bicapas, presentó el menor valor de pérdida de material. Las 
muestras con bicapas 1, 50 y 100 reducen la pérdida de masa en un $61 \%, 72 \%$ y $86 \%$, respectivamente en comparación con el sustrato sin recubrir, mostrando el efecto protector de la aplicación del sistema multicapas (Caicedo et al., 2012; Aperador et al 2011).

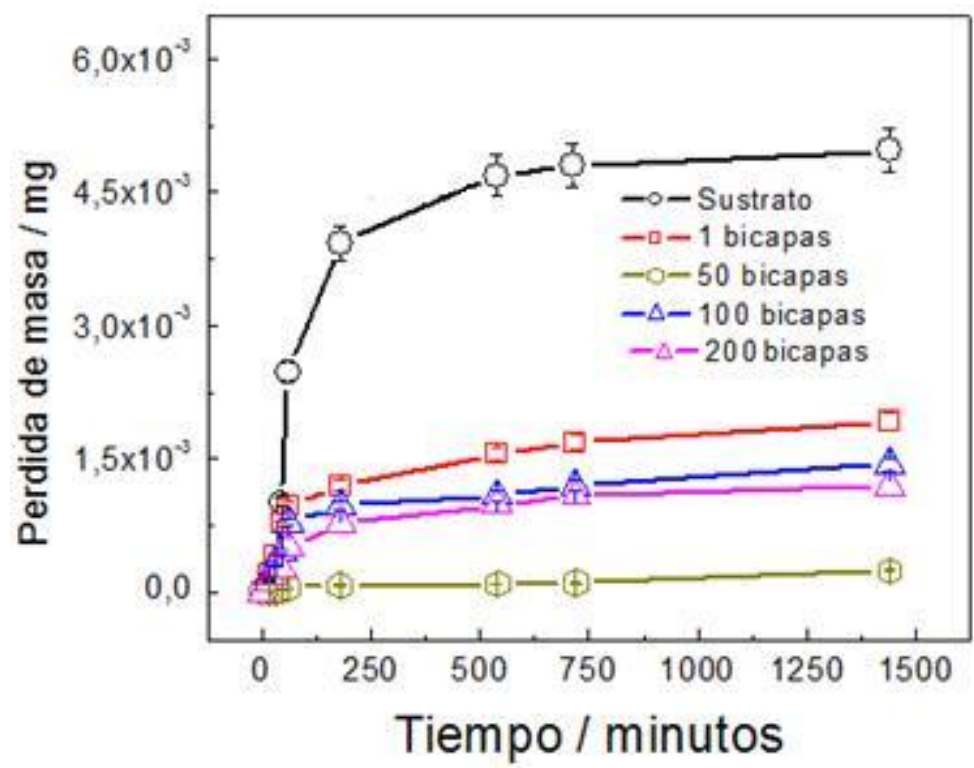

Fig. 3. Curvas dinámicas de desgaste ángulo erosión $90^{\circ}$ sin efecto corrosivo.

\section{Corrosión Dinámica}

La figura 4 muestra los diagramas de Nyquist del sustrato y los recubrimientos multicapas sumergidas en solución de $\mathrm{NaCl}$ al $0,5 \mathrm{M}$ y sometida a corrosión dinámica. Estos recubrimientos presentan un comportamiento de aumento de la impedancia total en cada uno de las multicapas evaluadas, siendo más prominente las bicapas 100 y 200. Los diagramas Nyquist correspondientes a las multicapas muestran un comportamiento capacitivo a elevadas frecuencias, en el que se define un semicírculo aplanado, este fenómeno de aplanamiento del semicírculo se asocia con un proceso de dispersión en la frecuencia, debido a que la superficie del electrodo no es homogénea. Adicionalmente, se observa un proceso de difusión que pretende definir un segundo semicírculo a bajas frecuencias.

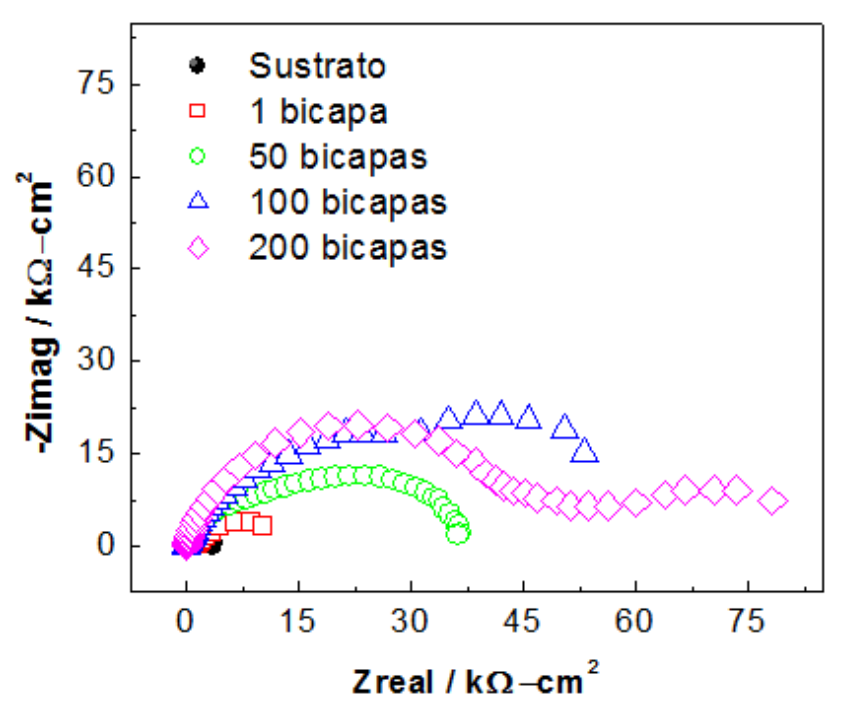

Fig. 4. Diagramas de impedancia de corrosión dinámica para bicapas de [TiN/ZrN] como función del número de bicapas.

En la tabla 1 se indican los valores de los parámetros utilizados en la simulación. Los valores de dichos parámetros se han obtenido mediante un programa no lineal de mínimos cuadrados complejos (CNLS). En la figura 5 se observa el circuito equivalente correspondiente a los diagramas de Nyquist para los 
recubrimientos, los cuales muestran una capacitancia denominada "elemento de fase constante" (CPE), que es independiente de reacciones farádicas, las cuales contribuyen con una pseudocapacitancia (CPE2+CPE1) a la impedancia total del sistema. Por otra parte, en esta celda electroquímica existe también una resistencia eléctrica, asociada a la resistencia del electrolito (Rs), que igualmente se hará manifiesta en impedancia total del sistema. Los valores de a (tabla 1), corresponde al coeficiente exponencial del corrimiento del ángulo de fase $(\Pi / 2)$; los valores de $\alpha$, para los recubrimientos y el sustrato para el CPE a altos valores de frecuencia, están en el rango de 0,69 y 0,74, indicando que la rugosidad de la superficie genera una distribución de carga. Para el CPE a bajas frecuencias muestra un valor de $\alpha$, de 0,72 para 1 bicapa y 0,76 para 50 bicapas, indicando migración o difusión de especies, para 100 y 200 bicapas se presentan valores de a de 0,89 y 0,91, generando distribución de la densidad de portadores de carga, es decir, una doble capa con estructura compleja.

Tabla 1. Comparación entre los datos experimentales correspondientes al sistema corrosión dinámica del sustrato y los recubrimientos con la variación de las bicapas.

\begin{tabular}{|l|c|c|c|c|c|c|c|}
\hline & $\begin{array}{c}\mathrm{R}_{\mathrm{s}} \\
\Omega \mathrm{cm}^{2}\end{array}$ & $\begin{array}{c}\mathrm{CPE}_{1} \\
\mu \mathrm{F} \mathrm{cm}^{-2} \mathrm{~s}^{-(1-\alpha 1)}\end{array}$ & $\alpha_{1}$ & $\begin{array}{c}\mathrm{R}_{1} \\
10^{3} \Omega \mathrm{cm}^{2}\end{array}$ & $\begin{array}{c}\mathrm{CPE}_{2} \\
\mu \mathrm{F} \mathrm{cm}^{-2} \mathrm{~s}^{-(1-\alpha 2)}\end{array}$ & $\alpha_{2}$ & $\begin{array}{c}\mathrm{R}_{2} \\
10^{3} \Omega \mathrm{cm}^{2}\end{array}$ \\
\hline 200 bicapas & $28,9(0,3 \%)$ & $5,42(1,6 \%)$ & $0,69(0,3 \%)$ & $46,3(3 \%)$ & $68,21(3 \%)$ & $0,91(0,4 \%)$ & $52,3(4 \%)$ \\
\hline 100 bicapas & $29,5(0,4 \%)$ & $3,32(2,4 \%)$ & $0,73(0,5 \%)$ & $31,6(2 \%)$ & $34,24(4 \%)$ & $0,89(0,6 \%)$ & $38,4(3 \%)$ \\
\hline 50 bicapas & $21,4(0,3 \%)$ & $3,06(2,3 \%)$ & $0,71(0,3 \%)$ & $15,8(3 \%)$ & $21,35(2 \%)$ & $0,76(0,9 \%)$ & $24,6(3 \%)$ \\
\hline 1 bicapa & $26,4(0,4 \%)$ & $2,86(1,4 \%)$ & $0,74(0,5 \%)$ & $3,05(5 \%)$ & $4,41(2 \%)$ & $0,72(0,4 \%)$ & $15,3(5 \%)$ \\
\hline Sustrato & $25,2(0,2 \%)$ & $2,08(1,6 \%)$ & $0,72(0,6 \%)$ & $0,91(5 \%)$ & & & \\
\hline
\end{tabular}

Para la muestra de acero 4140 (sustrato), se obtiene un pequeño semicírculo insignificante comparado con los resultados de los recubrimientos multicapas de [TiN/ZrN], el modelo que proporcionó mejor ajuste es el presentado en la figura 5a. En este circuito se observa una constante de tiempo conectada en paralelo con la resistencia a la transferencia de carga, y en serie con la resistencia de la solución. Para los recubrimientos variando las bicapas $(1,50,100$ y 200) se observan dos semicírculos (figura 4) por lo tanto se utilizó para modelar un circuito equivalente con dos elementos de fase constante (CPE1 y CPE2), representado en la figura $5 \mathrm{~b}$, los elementos CPE1-R1, se presentan a altas frecuencias y se asocian a las reacciones que ocurren alrededor de la capa de óxido superficial pasivante generado por los recubrimientos. Un segundo conjunto de elementos CPE2-R2, presentes a muy bajas frecuencias $(1 \mathrm{mHz})$ están relacionados con la capa barrera generada por la capa de óxido superficial y el sustrato debido a la transferencia de carga; este conjunto de elementos representa la respuesta de los procesos ocurridos en el sistema, los cuales son lentos en los recubrimientos obtenidos.

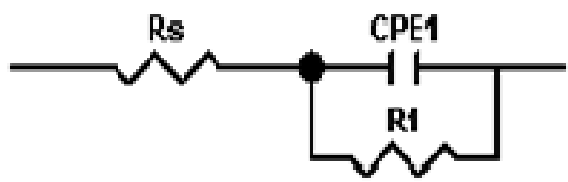

a)

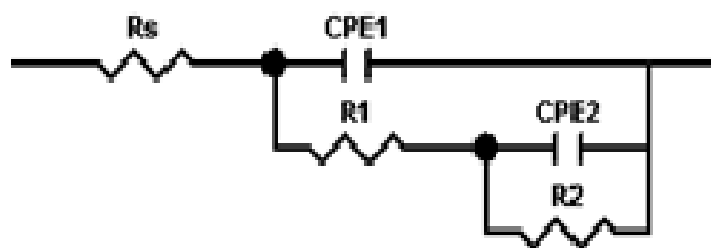

b)

Fig. 5. Circuito equivalente. a) circuito para ajustar los datos de impedancia del sustrato; b) Circuito para fitear los datos de impedancia de los recubrimientos con las modificaciones de las soluciones y número de bicapas.

\section{Corrosión-Erosión}

Los diagramas de Nyquist mostrados en la figura 6, presentan un comportamiento de disminución de la impedancia total en cada uno de las multicapas evaluadas en comparación con el efecto de la corrosión dinámica. El efecto combinado de la erosión y la corrosión resultó en una pérdida total de material elevada que es observada sumando los efectos aditivos de cada proceso por separado, lo cual indica que hay un sinergismo en los dos procesos. La componente sinérgica es la parte del daño total resultado de la 
interacción de la corrosión y el proceso de desgaste (Tabla 2). Los efectos combinados de desgaste y corrosión, ocasionaron mayor daño al causado por los efectos aditivos de cada uno, siendo más prominente para el sustrato AISI 4140 y multicapas con 1 bicapa. Al correlacionar el comportamiento de las demás multicapas, se encuentra que prácticamente los materiales de estudio presentan impedancia total similar para las multicapas correspondientes a 50 y 100 bicapas (Tabla 2).

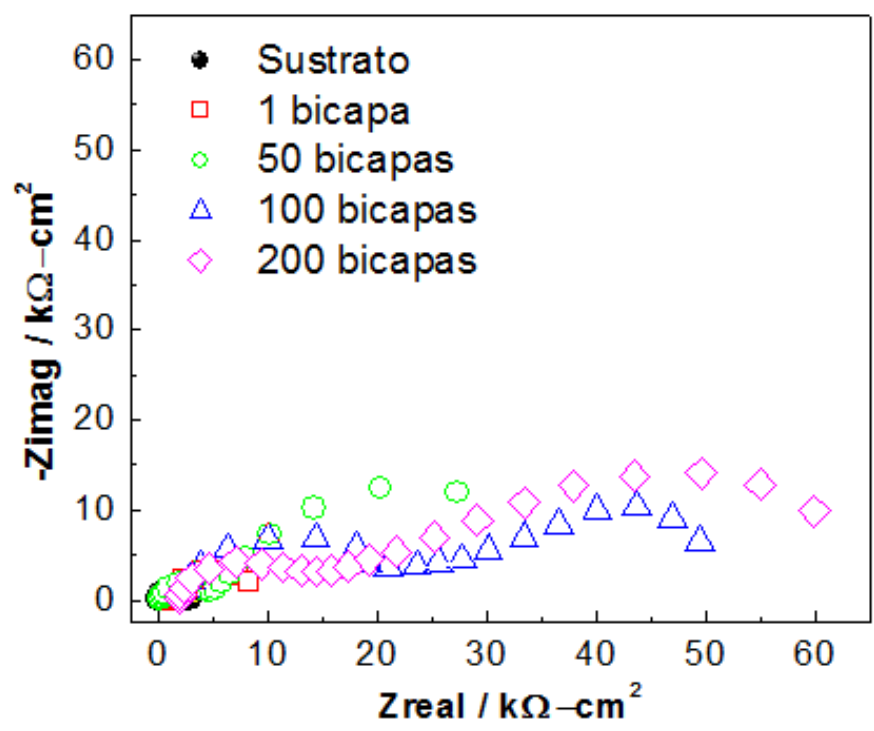

Fig. 6. Diagramas de impedancia de corrosión erosión para bicapas de [TiN/ZrN] como función del número de bicapas.

Tabla 2. Comparación entre los datos experimentales correspondientes al sistema corrosión erosión del sustrato y los recubrimientos.

\begin{tabular}{lccccccc}
\hline & $\begin{array}{c}\mathrm{R}_{\mathrm{s}} \\
\Omega \mathrm{cm}^{2}\end{array}$ & $\begin{array}{c}\mathrm{CPE}_{1} \\
\mu \mathrm{F} \mathrm{cm} \mathrm{cm}^{-2}\end{array}$ & $\alpha_{1}$ & $\begin{array}{c}\mathrm{R}_{1} \\
10^{3} \Omega \mathrm{cm}^{2}\end{array}$ & $\begin{array}{c}\mathrm{CPE}_{2} \\
\mu \mathrm{F} \mathrm{cm}^{-2} \mathrm{~s}^{-(1-\square \square}\end{array}$ & $\alpha \square$ & $\begin{array}{c}\mathrm{R}_{2} \\
10^{3} \Omega \mathrm{cm}^{2}\end{array}$ \\
\hline 200 bicapas & $22,11(0,4 \%)$ & $28,21(2,1 \%)$ & $0,74(0,3 \%)$ & $46,2(2 \%)$ & $54,12(3 \%)$ & $0,83(0,4 \%)$ & $52,12(5 \%)$ \\
\hline 100 bicapas & $32,2(0,3 \%)$ & $16,43(1,8 \%)$ & $0,72(0,2 \%)$ & $31,6(3 \%)$ & $29,15(2 \%)$ & $0,79(0,6 \%)$ & $38,4(4 \%)$ \\
\hline 50 bicapas & $34,2(0,3 \%)$ & $14,37(2,1 \%)$ & $0,74(0,3 \%)$ & $15,2(2 \%)$ & $19,13(3 \%)$ & $0,81(0,7 \%)$ & $34,6(3 \%)$ \\
\hline 1 bicapa & $29,6(0,6 \%)$ & $13,97(3,1 \%)$ & $0,71(0,3 \%)$ & $3,2(3 \%)$ & $3,12(2 \%)$ & $0,77(1,1 \%)$ & $16,2(3 \%)$ \\
\hline Sustrato & $31,4(0,5 \%)$ & $1,29(1,5 \%)$ & $0,76(0,2 \%)$ & $0,78(4 \%)$ & & & \\
\hline
\end{tabular}

\section{Sinergia Corrosión-Erosión}

En la figura 7 se observan los cálculos de sinergia que permiten establecer como las dos condiciones esenciales del desgate (mecánico y electroquímico) interactúan entre sí, ya sea para atenuar o intensificar uno de los dos mecanismos o para contribuir en el proceso global de desgaste de los materiales. Al observar la magnitud del sinergismo para cada uno de los materiales de estudio se observa que el desempeño de los mecanismos de desgaste esta implicitamente relacionado con la agresividad o efecto del medio sobre el material. Realizando un análisis global del desempeño de los mecanismos en medio corrosivo se observa que el material con mayor efecto sinergético es el acero AISI 4140, mientras que las multicapas presentan una disminución en función del aumento de las bicapas.

Este efecto se debe a la acción del ión cloruro, ya que la depolarización generada en el sistema promueve fenómenos de corrosión localizada que avanzan hacia la profundidad del material sin necesidad de ampliar las zonas afectadas, lo cuál es trabajo de la remoción mecánica por impacto. Este resultado indica que los micromecasnismos de desgaste son aquellos responsables de la presencia y avance de fenómenos de corrosión localizada y que favorecen una mayor remoción de las capas externas del material. Al obtener valores positivos en la sinergia evaluada se puede afirmar que la correlación en todos los casos es aditiva, es decir, la corrosión controla el deterioro del material y no el desgaste mecánico (ASTM 119 - 93). 


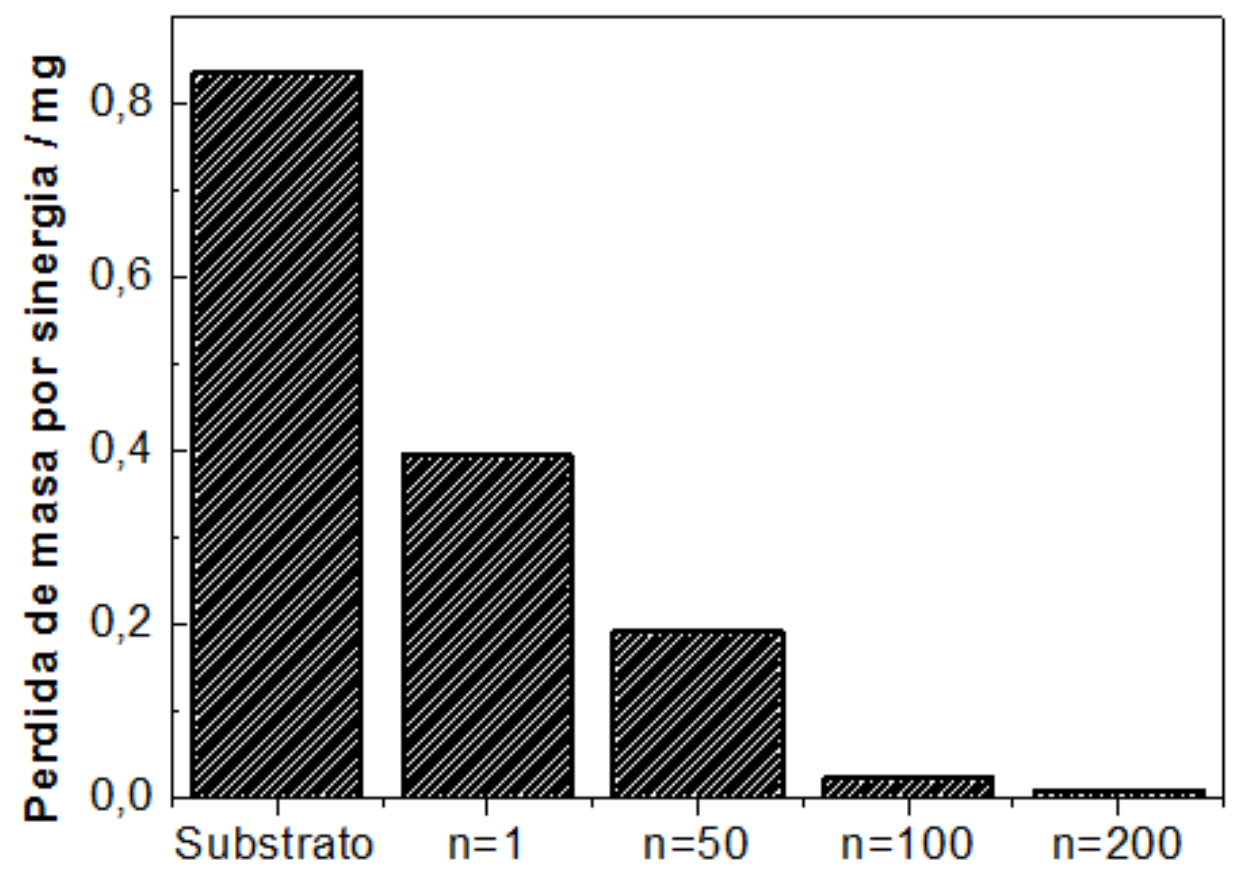

Fig. 7: Calculo de la sinergia debido al efecto aditivo de la corrosión para el sustrato y las bicapas de [TiN/ZrN].

\section{Morfología de la Degradación}

En la figura 8 se observan las micrografías de la superficie del sustrato y los recubrimientos de [TiN/ZrN] con $1,50,100$ y 200 bicapas luego del proceso de corrosión erosión a un ángulo de impacto de $90^{\circ}$. En la figura 8a se observa que parte del sustrato ha sido deteriorado debido al efecto de la corrosión dinámica, se observa una superficie con amplias cavidades bajo ángulo normal, esta superficie es resultado de la interacción de ambos medios de desgaste (electroquímico y mecánico) generando una mayor pérdida del material observado se relaciona con la remoción de material metálico por fatiga de las zonas adyacentes a las zonas anódicas.

En las figuras $8 b, 8 c, 8 d$ y $8 e$ en las bicapas de $n=1,50$ y 100 se observa el fenómeno erosivo, se refleja en una superficie rugosa y una leve textura en la dirección de impacto de las partículas principalmente para los recubrimientos con $n=1$ y 50 bicapas, este desgaste electroquímico del material trae consigo una superficie con claros signos de deterioro mecánico, en donde se observan huellas más profundas y una mayor rugosidad de la superficie, mientras que las bicapas $n=100$ y 200 se hace más notable el cambio de coloración de la superficie, debido al proceso electroquímico.

\section{CONCLUSIONES}

Los resultados muestran que el buen rendimiento de los sistemas multicapa en sistemas dinámicos de corrosión y corrosión por erosión es debido al hecho de que se aumenta la resistencia a la corrosión, generando un aumento frente a fenómenos de corrosión al aumentar el número de bicapas.

La sinergia de los mecanismos corrosión erosión, demostraron una acción positiva, indicando que el deterioro electroquímico es el que controla el desgaste del material y no el desgaste mecánico, esto fue corroborado con la microscopia electrónica de barrido realizada posterior a la acción corrosiva erosiva, ya que los recubrimientos con $n=100$ y 200, el deterioro fue causado por el medio corrosivo.

El comportamiento de los sistema multicapas en condiciones de erosión, revelan que los recubrimientos generan una disminución en la perdida de material, y es más significativo para el sistema multicapa con $\mathrm{n}=200$, es decir, el efecto erosivo en el sistema de multicapas permitió establecer buenas propiedades mecánicas generando absorción y distribución de la energía de impacto.

A través del análisis microscópico, se observa que el sistema de multicapas produce un efecto protector al sustrato, cuando están sometidas a la sinergia de corrosión-erosión. En las muestras recubiertas se observa el efecto nocivo generado por la acción de la erosión generado por la energía de impacto de las partículas abrasivas de sílice, y zonas en donde el efecto protector genera una baja rugosidad. 


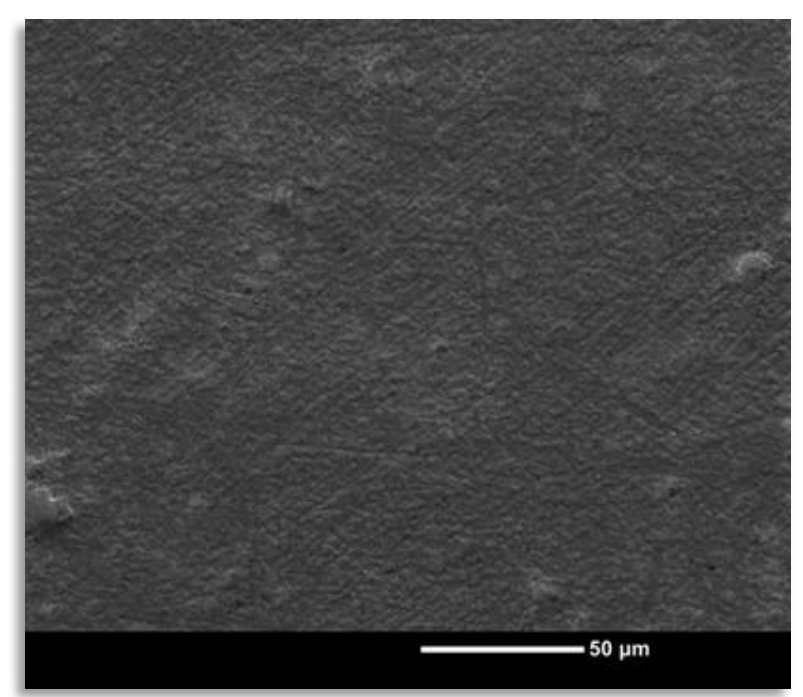

(a)

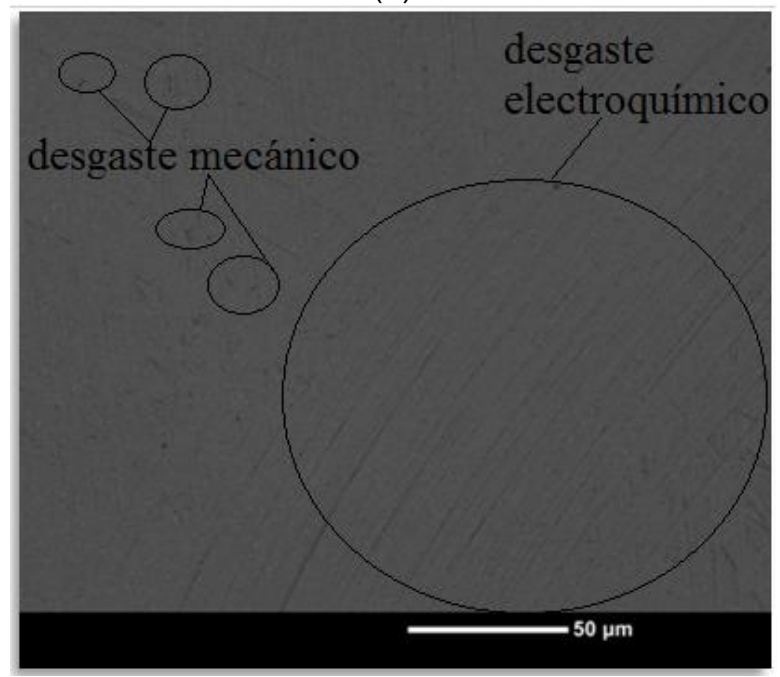

(c)

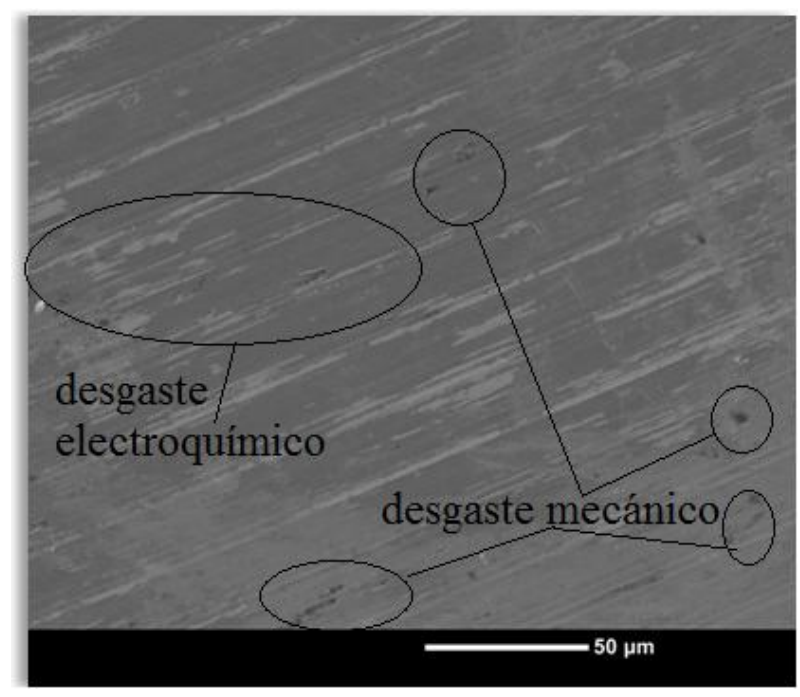

(b)

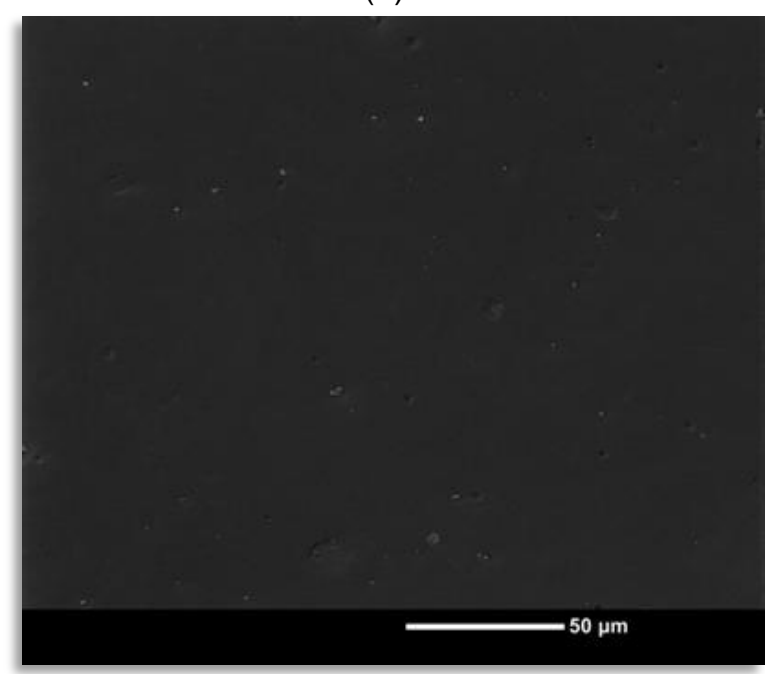

(d)

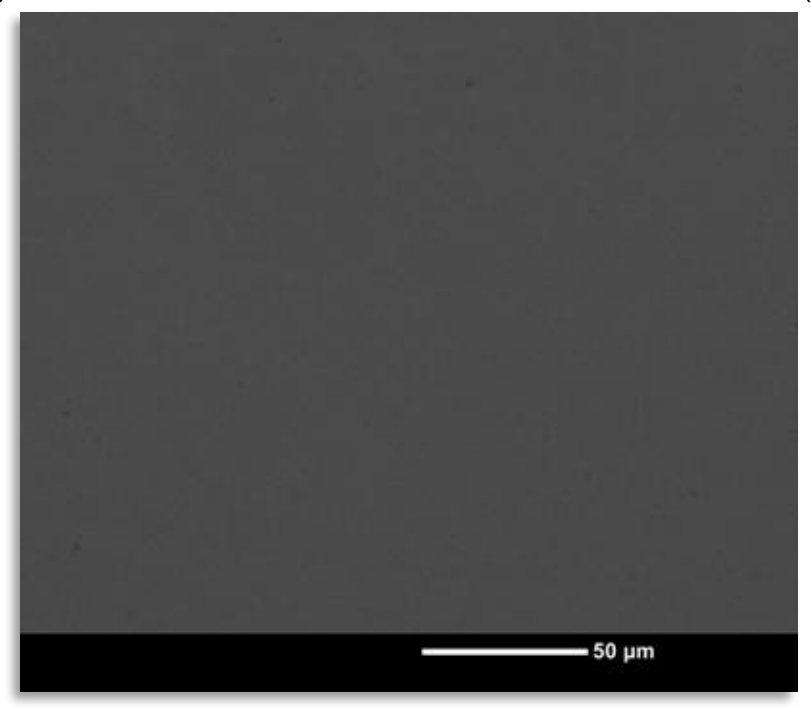

(e)

Fig. 8. Micrografía de SEM para muestras sometidas a corrosión - erosión. a) Sustrato; b) recubrimiento de [TiN/ZrN] con $n=1$; c) $n=50$; d) $n=100$ y e) $n=200$. 


\section{REFERENCIAS}

Aperador,W., y otros autores. Bilayer period effect on corrosion-erosion resistance for [TiN/AlTiN]n multilayered growth on AISI 1045 steel, Journal of Physics and Chemistry of Solids., 71, (12), 1754-1759 (2010).

Aperador, W., Ramírez-Martínez C., Bautista Ruiz J. Sinergia entre la corrosión erosión del acero 1045 recubierto por multicapas de TiN/TiAIN. Revista Mexicana De Física , 57, (4), 350-355, (2011).

ASTM 119 - 93. Standard Guide for Determining Synergism Between Wear and Corrosion. West Conshohocken, PA. American Society for Testing and Materials, 2009.

ASTM G3 - 89. Standard Practice for Conventions Applicable to Electrochemical Measurements in Corrosion Testing, West Conshohocken, PA, American Society for Testing and Materials, 2010.

Barshilia, H. y otros autores. Growth and characterization of TiAIN/CrAIN superlattices prepared by reactive direct current magnetron sputtering, Journal of Vacuum Science \& Technology A: Vacuum, Surfaces, and Films, 27, (1), 29-36 (2009).

Barshilia, Harish C. Deepthi, B. Selvakumar, N. Jain, Anjana Rajam, K.S. Nanolayered multilayer coatings of CrN/CrAIN prepared by reactive DC magnetron sputtering, Applied Surface Science, 253, (11), 5076-5083 (2007).

Caicedo J. C., Cabrera G., Aperador W. Escobar C., Amaya C. Corrosion-Erosion Effect on TiN/TiAIN Multilayers, Journal of materials engineering and performance. 21, (9), 1949-1955, (2012).

Caicedo, J. C, y otros autores. Effect of applied bias voltage on corrosion-resistance for TiC1-xNx and Ti1-xNbxC1-yNy coatings, Applied Surface Science, 256, (9), 2876-2883 (2010).

Farzad Mohammadi, Jingli Luo, Effect of cold work on erosion-corrosion of 304 stainless steel, Corrosion Science, 53, (2), 549-556 (2011).

Junhua, X., Geyang, L., Mingyuan, G. The microstructure and mechanical properties of TaN/TiN and TaWN/TiN superlattice films, Thin Solid

López D. Neusa A. Falleiros A. Tschiptschin A.P. Effect of nitrogen on the corrosion-erosion synergism in an austenitic stainless steel, Tribology International, 44, (5), 610-616 (2011).

Neville A., Wang C., Erosion-corrosion of engineering steels-Can it be managed by use of chemicals?, Wear, 267, (11), 2018-2026 (2009).

Nieto J., y otros autores. Evaluación de la influencia del voltaje bias sobre la resistencia a la corrosión de películas delgadas de AINbN, Dyna, Año 77, (162), 161-168 (2010).

Philip C. Yashar, William D. Sproul, Nanometer scale multilayered hard coatings, Vacuum.Vol. 55, (3), 179190 (1999).

Rajahram S.S., Harvey T.J., Wood R.J.K., Evaluation of a semi-empirical model in predicting erosioncorrosion, Wear, 267, (11), 1883-1893 (2009).

Xi Chu y Scott Barnett A., Model of superlattice yield stress and hardness enhancements, Journal of Applied Physics, 77 (9), 77, $4403-4412$ (1995). 